\title{
Quasi sure quadratic variation of smooth martingales
}

\author{
By \\ Jiagang REN*
}

\section{Introduction}

Suppose that $\boldsymbol{M}=\left\{M_{t}, t \in I\right\}$ is a continuous $L^{p}$-martingale $(p \geq 2)$, where $I$ is an interval of $\boldsymbol{R}_{+}=[0, \infty)$ (may be $\boldsymbol{R}_{+}$itself). By the well-known DoobMeyer decomposition theorem, there exists a unique increasing process $\langle\boldsymbol{M}\rangle$ $=\left\{\langle M\rangle_{t}, t \in I\right\}$ such that $\boldsymbol{M}^{2}-\langle\boldsymbol{M}\rangle$ is a continuous $L^{p / 2}$-martingale. Moreover, P. W. Millar [12] and D. Nualart [13] showed that the process $\langle\boldsymbol{M}\rangle$ can be obtained as the $L^{p / 2}$-limit of sums of the form $\Sigma M\left(\Delta_{i}\right)$, where $\left\{\Delta_{i}\right\}$ is a subdivision of the interval $I$, as $\max _{i}\left|\Delta_{i}\right| \rightarrow 0$.

In the present paper we propose to study the quasi sure properties of the quadratic variation of smooth martingales, a notion introduced recently by $\mathrm{P}$. Malliavin and D. Nualart [9]. We shall prove that the process of the quadratic variation of a smooth martingale admits an $\infty$-modification, which can be constructed as the quasi sure limit of sums of the form $\sum M\left(\Delta_{i}\right)$. Our tool is the quasi sure version of Kolmogorov's criterion for the continuity of trajectories of stochastic processes (cf. [17]). Necessary estimations which enable us to apply this criterion will be obtained. This makes the subject of section 3. In section 4 we will be able to extend the results of section 3 to the case of two-parameter smooth martingales. At last in section 5 we discuss possible extensions and applications. We prove, in particular, that the quadratic variation of the Brownian motion is quasi surely $t$.

The main results of this paper were announced in [21].

\section{Preliminaries}

Now let us recall and fix some notations and notions. We shall work on the probability space $(X, H, \mu)$, where $X$ is the space of continuous maps from $[0,1]$ to $\boldsymbol{R}^{d}$, null at zero; $H$ is the usual Cameron-Martin subspace and $\mu$ the standard Wiener measure. Denote by $W_{2 r}^{p}$ the Sobolev space of order $2 r$ and of power $p$ over $X$ and $W_{\infty}$ their intersection over indexes $p>1$ and $r$ $\geq 0$. For any natural number $r$, two equivalent norms in $W_{2 r}^{p}$ are defined

*JSPS Program; Supported also by Fok Ying Tong Education Foundation and NSF of China Communicated by Prof. S. Watanabe, February 1, 1993 
respectively by

$$
\|F\|_{p, 2 r}=\left\|(I-L)^{r} F\right\|_{p}
$$

and

$$
\|F\|_{p, 2 r}^{\prime}=\left\|\nabla^{2 r} F\right\|_{p}+\|F\|_{p}
$$

where $L$ is the Ornstein-Uhlenbeck operator and $D$ the gradient operator (cf [8], [20]). Given an open set $O$ of $X$, its $(p, r)$-capacity is defined by (cf [7])

$$
C_{p, r}(O)=\inf \left(\|F\|_{p, 2 r} ; F \geq 0, F \geq 1 \quad \mu \text {-a.e. on } O\right) \text {; }
$$

and for any subset $A$ of $X$, by

$$
C_{p, r}(A)=\inf \left(C_{p, r}(O) ; O \text { open and } A \subset O\right) .
$$

Let $\left\{w_{s}, 0 \leq s \leq 1\right\}$ be the $d$-dimensional Brownian motion realized by the coordinate process on $(X, H, \mu)$ and $\mathscr{F}_{t}$ the $\sigma$-algebra generated by $\left\{w_{s}, 0 \leq s\right.$ $\leq t\}$. Then any $\left\{\mathscr{F}_{t}\right\}$-continuous square integrable martingale $\boldsymbol{M}=\left\{M_{t}, 0 \leq t\right.$ $\leq 1$ \} can be represented as a stochastic integral

$$
M_{t}=\sum_{i=1}^{d} \int_{0}^{t} f_{s}^{i} d w_{s}^{i}, \quad 0 \leq t \leq 1 .
$$

Following P. Malliavin and D. Nualart, we say that $\boldsymbol{M}$ is smooth if the following condition is fulfiled:

$$
\begin{aligned}
& f_{s} \in W_{\infty}\left(\boldsymbol{R}^{d}\right) \text { for almost all } 0 \leq s \leq 1 \\
& \text { and } \int_{0}^{1}\left\|f_{s}^{i}\right\|_{p, 2 r}^{p} d s<\infty \text { for all } p, r .
\end{aligned}
$$

(Note that we do not impose in the definition the condition guaranteeing the quasi sure convergence in [9], since we work now on a finite time interval instead of $\boldsymbol{R}_{+}$.)

\section{Main results}

Let $\boldsymbol{M}$ be a smooth martingale represented as (1). Then by [9, Theorem 4.2], $\boldsymbol{M}$ admits an $\infty$-modification, which will be denoted still by $\boldsymbol{M}$ for notational simplicity. It is well known that the process of quadratic variation of $\boldsymbol{M}$ is given by

$$
\langle M\rangle_{t}=\sum_{i=1}^{d} \int_{0}^{t} f_{s}^{i^{2}} d s, \quad 0 \leq t \leq 1 .
$$

At first we give

Theorem 3.1. $\langle M\rangle$ admits an $\infty$-modification. 
Proof. By [16, Theorem 3.1 and lemma 4.1] (cf. also [17]), it is sufficient to prove the following two facts:

$$
\begin{aligned}
& \left\|\langle M\rangle_{t}-\langle M\rangle_{s}\right\|_{p}^{p} \leq C|t-s|^{p-1}, \quad p \geq 2,0 \leq s, t \leq 1 . \\
& \langle M\rangle_{t} \in W_{\infty}, \quad \forall t \in[0,1] \text { and } \sup _{0 \leq t \leq 1}\left\|\langle M\rangle_{t}\right\|_{p, 2 r}<\infty \forall p, r .
\end{aligned}
$$

But they are both trivial from the expression (1), condition (C1) in section 1 and Stroock's commutation formula.

Q.E.D.

Denoting still by $\langle\boldsymbol{M}\rangle$ itself its $\infty$-modification, we can state the following

Theorem 3.2. The convergence:

$$
\lim _{n \rightarrow \infty} \sum_{i=0}^{2^{n}-1}\left(M_{t_{i+1}^{n} \wedge t}-M_{t_{i}^{n} \wedge t}\right)^{2}=\langle M\rangle_{t}
$$

holds uniformly in $t \in[0,1]$, q.s., where $t_{i}^{n}=i 2^{-n}$.

Proof. To avoid surcharging the calculus, in the sequel all the constants depending only on $M, p$ and $d$, but not on $n$ and the parameters $s, t, \cdots$, will be simply denoted by $C$. Put

$$
X\left(2^{-n}, t\right)=\sum_{i=0}^{2^{n}-1}\left(M_{t_{i+1}^{n} \wedge t}-M_{t_{i}^{n} \wedge t}\right)^{2},
$$

and

$$
X(s, t)= \begin{cases}X\left(2^{-n}, t\right)+\left(s-2^{-n}\right)\left(2^{-n}-2^{-(n+1)}\right)^{-1}\left(X\left(2^{-n}, t\right)-X\left(2^{-(n+1)}, t\right)\right) \\ \text { if } 2^{-(n+1)} \leq s \leq 2^{-n}, \\ \sum_{i=1}^{d} \int_{0}^{t} f_{u}^{i^{2}} d u & \text { if } s=0 .\end{cases}
$$

Then it is sufficient to prove that $X(s, t)$ admits an $\infty$-modification. By the quasi sure version of Kolmogorov's criterion (cf. [16, Theorem 3.1]; also [17] and [18]) and [16, Lemma 4.1], and taking (2) into account, we need only to prove the following inequalities:

$$
\begin{aligned}
& \left\|X(s, t)-X\left(s^{\prime}, t^{\prime}\right)\right\|_{p}^{p} \leq C\left[\left|s-s^{\prime}\right|^{\frac{(p-1)}{2}}+\left|t-t^{\prime}\right|^{\frac{p-1}{2}}\right], \quad \forall p . \\
& \sup _{s, t}\|X(s, t)\|_{p, 2 r}<\infty, \quad \forall p .
\end{aligned}
$$

We do the proof in two steps.

Proof of (3). Since it is trivial to see that

$$
\left\|X(0, t)-X\left(0, t^{\prime}\right)\right\|_{p}^{p} \leq C\left|t-t^{\prime}\right|^{p-1},
$$


it is enough to prove that (note that $X$ is piecewise linear in $s$ )

$$
\sup _{n}\left\|X\left(2^{-n}, t\right)-X\left(2^{-n}, t^{\prime}\right)\right\|_{p}^{p} \leq C\left|t-t^{\prime}\right|^{\frac{p-1}{2}} .
$$

and

$$
\sup _{t}\left\|X\left(2^{-n}, t\right)-X(0, t)\right\|_{p}^{p} \leq C 2^{-\frac{n}{2}(p-1)}
$$

We first look at (5). Put $t_{n}=\frac{\left[2^{n} t\right]}{2^{n}}, t_{n}{ }^{+}=\frac{\left[2^{n} t\right]+1}{2^{n}}$, where [s] denotes the maximal integer not bigger than $s$. We can of course assume $t<t^{\prime}$. We will discuss in the following two cases.

(A) The case $t_{n}=t_{n}^{\prime}$. In this case we have

$$
\begin{aligned}
& \left\|X\left(2^{-n}, t\right)-X\left(2^{-n}, t^{\prime}\right)\right\|_{p}^{p} \\
& \quad=\left\|\left(M_{t}-M_{t_{n}}\right)^{2}-\left(M_{t^{\prime}}-M_{t_{n}}\right)^{2}\right\|_{p}^{p} \\
& \quad=\left\|\left(M_{t}-M_{t^{\prime}}\right)\left(M_{t}+M_{t^{\prime}}-2 M_{t_{n}}\right)\right\|_{p}^{p} \\
& \quad \leq C\left\|\left(M_{t}-M_{t^{\prime}}\right)\right\|_{2 p}^{p}\left\|_{t}+M_{t^{\prime}}-2 M_{t_{n}}\right\|_{2 p}^{p} \\
& \quad \leq C\left\|\sum_{j=1}^{d} \int_{t}^{t^{\prime}} f_{u}^{j} d w_{u^{j}}^{j}\right\|_{2 p}^{p} \\
& \quad \leq C \sum_{j=1}^{d}\left\{E\left(\int_{t}^{t^{\prime}}\left|f_{u^{i}}\right|^{2} d u\right)^{p}\right\}^{1 / 2} \quad \text { (by Burkhoder's inequality) } \\
& \quad \leq C\left|t-t^{\prime}\right|^{\frac{p-1}{2}} .
\end{aligned}
$$

(B) The case $t_{n}<t_{n}^{\prime}$. In this case we have

$$
\begin{aligned}
& \left\|X\left(2^{-n}, t^{\prime}\right)-X\left(2^{-n}, t\right)\right\|_{p}^{p} \\
& =E\left|\left(M_{t_{n^{+}}}-M_{t_{n}}\right)^{2}-\left(M_{t}-M_{t_{n}}\right)^{2}+\sum_{i=t_{n^{+} 2^{n}}}^{t_{\prime^{\prime} 2^{n}}}\left(M_{t_{i+1}^{n} \wedge t^{\prime}}-M_{t_{i} n \wedge t^{\prime}}\right)^{2}\right|^{p} \\
& \leq C E\left|\left(M_{t_{n^{+}}}-M_{t}\right)\left(M_{t_{n^{+}}}+M_{t}-2 M_{t_{n}}\right)\right|^{p}+C E\left(\sum_{i=t_{n^{+} 2^{n}}}^{t_{n^{\prime} 2^{n}}}\left(M_{t_{i+1}^{n} \wedge t^{\prime}}-M_{t_{i^{n}} \wedge t^{\prime}}\right)^{2}\right)^{p} .
\end{aligned}
$$

The first term of the RHS can be estimated in the same way as in the case (A), and is bounded by $\left|t_{n}{ }^{+}-t\right|^{\frac{p-1}{2}}$. The last term can be estimated by using Burkholder's inequality for martingales with both discrete and continuous parameter, and in both ways:

$$
E\left(\sum_{i=t_{n}+2^{n}}^{t_{n^{\prime} 2^{n}}}\left(M_{t^{n}+1 \wedge t^{\prime}}-M_{t_{i} n \wedge t^{\prime}}\right)^{2}\right)^{p}
$$




$$
\begin{aligned}
& \leq C E\left|\sum_{i=t_{n^{+} 2^{n}}}^{t_{n^{\prime}} 2^{n}}\left(M_{t_{i+1}^{n} \wedge t^{\prime}}-M_{t_{i} n \wedge t^{\prime}}\right)\right|^{2 p} \\
& =C E\left|\int_{t_{n^{+}}}^{t_{n^{\prime}}} \sum_{j=1}^{d} f_{u}^{j} d w_{u}^{j}\right|^{2 p} \\
& \leq\left.\left. C \sum_{j=1}^{d} E\left|\int_{t_{n^{+}}}^{t^{\prime}} \sum_{j=1}^{d}\right| f_{u}^{j}\right|^{2} d u\right|^{p} \\
& \leq C\left|t^{\prime}-t_{n}{ }^{+}\right|^{p-1} .
\end{aligned}
$$

Hence

$$
\begin{aligned}
\left\|X\left(2^{-n}, t^{\prime}\right)-X\left(2^{-n}, t\right)\right\|_{p}^{p} & \leq C\left[\left|t_{n}^{+}-t\right|^{\frac{p-1}{2}}+\left|t^{\prime}-t_{n}^{+}\right|^{p-1}\right] \\
& \leq C\left|t^{\prime}-t\right|^{\frac{p-1}{2}} .
\end{aligned}
$$

Combining case (A) and case (B) implies (5). For (6) we have:

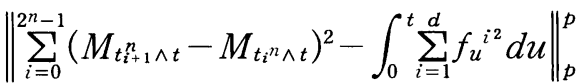

$$
\begin{aligned}
& =\left\|\sum_{i=0}^{2 n-1}\left(M_{t_{i+1}^{n} \wedge t}-M_{t_{i^{n}} \wedge t}\right)^{2}-\int_{t_{i} n \wedge t}^{t_{i+1}^{n} \wedge t} \sum_{i=1}^{d} f_{u^{i 2}} d u\right\|_{p}^{p} \\
& =\left\|\sum_{i=0}^{2^{n}-1} \sum_{j=0}^{d} \int_{t^{n}{ }^{n} \wedge t}^{t_{i+1}^{n} \wedge t}\left(M_{u}-M_{t_{i}{ }^{n} \wedge t}\right) f_{u}^{j} d w_{u}^{j}\right\|_{p}^{p} \text { (by Ito's formula) } \\
& \leq C \sum_{j=0}^{d}\left\|\sum_{i=0}^{2^{n}-1} \int_{t_{i}^{n} \wedge t}^{t_{i+1}^{n} \wedge t}\left(M_{u}-M_{t_{i}^{n} \wedge t}\right) f_{u}^{j} d w_{u}^{j}\right\|_{p}^{p} \\
& \leq C \sum_{j=0}^{d} E\left|\sum_{i=0}^{2^{n}-1}\left(\int_{t_{i}^{n} \wedge t}^{t_{i+1}^{n} \wedge t}\left(M_{u}-M_{t_{i}^{n} \wedge t}\right) f_{u}^{j} d w_{u}^{j}\right)^{2}\right|^{p / 2}
\end{aligned}
$$

(by Burkholder's inequality for martingales with discrete parameter)

$$
\leq C 2^{n\left(\frac{p}{2}-1\right)} \sum_{j=1}^{d} E^{2^{n}-1}\left|\int_{i=0}^{t_{i+1}^{n} \wedge t}\left(M_{t^{n} \wedge t}-M_{t_{i}^{n} \wedge t}\right) f_{u}^{j} d w_{u}^{j}\right|^{p}
$$

(by Hölder's inequality)

$$
\leq C 2^{n\left(\frac{p}{2}-1\right)} \sum_{j=1}^{d} \sum_{i=0}^{2^{n}-1} E\left(\int_{t^{n} \wedge t}^{t_{i+1}^{n} \wedge t}\left(M_{u}-M_{t_{i} n \wedge t}\right)^{2}\left|f_{u}^{j}\right|^{2} d u\right)^{p / 2}
$$

(by Burkholder's inequality for martingales with continuous parameter)

$$
\leq C 2^{n\left(\frac{p}{2}-1\right)} 2^{-n\left(\frac{p}{2}-1\right)} \sum_{j=1}^{d} \sum_{i=0}^{2^{n}-1} \int_{t^{n} \wedge t}^{t_{i+1}^{n} \wedge t} E\left|M_{u}-M_{t_{i}{ }^{n} \wedge t}\right|^{p}\left|f_{u^{j}}\right|^{p} d u
$$

(by Hölder's inequality) 


$$
\leq C \sum_{j=1}^{d} \sum_{i=0}^{2^{n}-1} \int_{t_{i} n \wedge t}^{t_{i+1}^{n} \wedge t}\left(E\left|M_{u}-M_{t_{i} n \wedge t}\right|^{2 p}\right)^{1 / 2}\left(E\left|f_{u}^{j}\right|^{2 p}\right)^{1 / 2} d u
$$

(by Hölder's inequality)

But for $u \in\left[t_{i}^{n} \wedge t, t_{i+1}^{n} \wedge t\right]$, we have

(8)

$$
\begin{aligned}
& E\left|M_{u}-M_{t_{i} n \wedge t}\right|^{2 p} \\
& =E\left|\sum_{j=1}^{d} \int_{t_{i}{ }^{n} \wedge t}^{u} f_{v}^{j} d w_{v}^{j}\right|^{2 p} \\
& \quad \leq C \sum_{j=1}^{d}\left(\int_{t_{i}^{n} \wedge t}^{t_{i+1}^{n} \wedge t}\left|f_{v}^{j}\right|^{2} d v\right)^{p} \\
& \quad \leq C 2^{-\frac{n}{2}(p-1)} \sum_{j=1}^{d} \int_{t_{i}{ }^{n} \wedge t}^{t_{i+1}^{n} \wedge t}\left|f_{v}{ }^{j}\right|^{2 p} d v \\
& \quad \leq C 2^{-\frac{n}{2}(p-1)} .
\end{aligned}
$$

Substituting (8) into (7) and taking (C1) into account, we get

$$
\begin{aligned}
& \left\|_{i=0}^{2^{n}-1}\left(M_{t_{i+1}^{n} \wedge t}-M_{t_{i}{ }^{n} \wedge t}\right)^{2}-\int_{0}^{t} \sum_{i=1}^{d} f_{u}^{i 2} d u\right\|_{p}^{p} \\
& \quad \leq C 2^{-\frac{n}{2}(p-1)} \sum_{j=1}^{d} \sum_{i=0}^{2^{n}-1} \int_{t_{i}{ }^{n} \wedge t}^{t_{i+1}^{n} \wedge t}\left(E\left|f_{s}^{j}\right|^{2 p}\right)^{1 / 2} d s \\
& \quad \leq C 2^{-\frac{n}{2}(p-1)} \sum_{j=1}^{d} \int_{0}^{1}\left(E\left|f_{s}^{j}\right|^{2 p}\right)^{1 / 2} d s \\
& \quad \leq C 2^{-\frac{n}{2}(p-1)} \sum_{j=1}^{d}\left(\int_{0}^{1} E\left|f_{s}^{j}\right|^{2 p} d s\right)^{1 / 2} \\
& \quad \leq C 2^{-\frac{n}{2}(p-1)}
\end{aligned}
$$

as desired. (6) is thus established.

Proof of (4) To prove (4) we need the following result which is easily deduced from [6, lemma 2.2].

Proposition 3.1. Let $f_{u}^{j} \in W_{\infty}(\boldsymbol{R})(j=1, \cdots, d, u \in[0,1])$ and define

$$
M_{t}=\sum_{j=1}^{d} \int_{0}^{t} f_{u}^{j} d w_{u}^{j}
$$

Put $f_{u}=\left(f_{u}{ }^{1}, \cdots, f_{u}{ }^{n}\right)$. Then we have

$$
\nabla^{n} M_{t}=\sum_{j=1}^{d} \int_{0}^{t} \nabla^{n} f_{u}^{j} d w_{u}^{j}+n \int_{0}^{t \wedge \cdot} \nabla^{n-1} f_{u} d u, \quad n \geq 1 .
$$


Now we turn to the proof of (4). Since it is trivial that $\sup _{t}\left\|\int_{0}^{t} f_{u}^{2} d u\right\|_{p, 2 r}$ $<\infty$, it suffices to prove that

$$
\sup _{n, t}\left\|_{i=0}^{2^{n}-1}\left(M_{t_{i+1}^{n} \wedge t}-M_{t^{n} \wedge t}\right)^{2}\right\|_{p, 2 r}<\infty, \quad \forall p, r
$$

First we look at the derivative of the second order. By the chain rule of $L$ it follows that

$$
\begin{aligned}
& L\left(\sum_{i=0}^{2^{n}-1}\left(M_{t_{i+1}^{n} \wedge t}-M_{t^{n} \wedge t}\right)^{2}\right) \\
& \quad=\sum_{i=0}^{2^{n}-1} 2\left(M_{t_{i+1}^{n} \wedge t}-M_{t^{n} \wedge t}\right) L\left(M_{t_{i+1}^{n} \wedge t}-M_{t_{i}^{n} \wedge t}\right)+\left\|D\left(M_{t_{i+1}^{n} \wedge t}-M_{t_{i}^{n} \wedge t}\right)\right\|_{H}{ }^{2} \\
& \quad \triangleq I_{1}{ }^{n}+I_{2}{ }^{n}
\end{aligned}
$$

By [17, lemma 4.3], $L M$ is also an $L^{p}$-martingale. Hence we have by Millar [12] (cf. also [13]):

$$
\lim _{n \rightarrow \infty} \sup _{t} E\left|I_{1}{ }^{n}(t)\right|^{p}=\sup _{t} E\left|\langle M, L M\rangle_{t}\right|^{p}<\infty .
$$

Consequently

$$
\sup _{n, t} E\left|I_{1}^{n}(t)\right|^{p}<\infty .
$$

Now we deal with $I_{2}{ }^{n}$. From Proposition 3.1 we deduce that

$$
\nabla\left(M_{t_{i+1}^{n} \wedge t}-M_{t_{i}^{n} \wedge t}\right)=\sum_{j=1}^{d} \int_{t_{i}^{n} \wedge t}^{t_{i+1}^{n} \wedge t} \nabla f_{u}^{j} d w_{u}^{j}+\int_{t_{i}^{n} \wedge t \wedge}^{t_{i+1}^{n} \wedge t \wedge \cdot} f_{u} d u .
$$

Therefore

$$
\begin{aligned}
& \sum_{i=0}^{2^{n}-1}\left\|\nabla\left(M_{t_{i+1}^{n} \wedge t}-M_{t_{i} n \wedge t}\right)\right\|_{H}^{2} \\
& \quad \leq \sum_{j=1}^{d} \sum_{i=0}^{2^{n-1}}\left\|\int_{t_{i}{ }^{n} \wedge t}^{t_{i+1}^{n} \wedge t} \nabla f_{u}^{j} d w_{u}^{j}\right\|_{H}^{2}+\sum_{j=1}^{d} \sum_{i=0}^{2^{n}-1} \int_{t^{i} \wedge t}^{t_{i+1}^{n} \wedge t} f_{u}^{j^{2}} d u \\
& \quad \triangleq I_{21}^{n}+I_{22}^{n} .
\end{aligned}
$$

For $I_{21}^{n}$ we have

$$
E\left|I_{21}^{n}(t)\right|^{p} \leq \sum_{j=1}^{d} E\left(\sum_{i=0}^{2^{n}-1}\left\|\int_{t_{i}^{n} \wedge t}^{t_{i+1}^{n} \wedge t} \nabla f_{u}^{j} d w_{u}^{j}\right\|_{H}^{2}\right)^{p} .
$$

By Burkholder's inequality for Hilbert-valued martingales with discrete parameter it is bounded by 


$$
C \sum_{j=1}^{d} E\left(\sup _{i}\left\|\int_{0}^{t_{i+1}^{n} \wedge t} \nabla f_{u}^{j} d w_{u}^{j}\right\|_{H}\right)^{2 p} .
$$

Therefore (again by Burkholder's inequality, but in reverse way)

$$
\begin{aligned}
E\left|I_{21}^{n}(t)\right|^{p} & \leq C \sum_{j=1}^{d} E\left(\int_{0}^{t}\left\|\nabla f_{u^{j}}\right\|_{H}{ }^{2} d u\right)^{p} \\
& \leq C \sum_{j=1}^{d} E \int_{0}^{1}\left\|\nabla f_{u}^{j}\right\|_{H}{ }^{2 p} d u<\infty .
\end{aligned}
$$

And for $I_{22}^{n}$ we have

$$
\begin{aligned}
E\left|I_{22}^{n}\right|^{p} & =E\left(\int_{0}^{t} \sum_{j=1}^{d} f_{u^{j}}^{j^{2}} d u\right)^{p} \\
& \leq C \sum_{j=1}^{d} \int_{0}^{1} E\left|f_{u^{j}}\right|^{2 p} d u<\infty .
\end{aligned}
$$

A combination of (14), (15) and (16) yields

$$
\sup _{n, t} E\left(\sum_{i=0}^{2^{n}-1}\left\|\nabla\left(M_{t_{i+1}^{n} \wedge t}-M_{t_{i} n \wedge t}\right)\right\|_{H}^{2}\right)^{p}<\infty .
$$

Hence

$$
\sup _{n, t}\left\|\sum_{i=0}^{2^{n}-1}\left(M_{t_{i+1}^{n} \wedge t}-M_{t^{n} \wedge t}\right)^{2}\right\|_{p, 2}<\infty,
$$

proving (10) for $r=1$.

Now we proceed to estimat the derivative of the fourth order (i.e, $r=2$ in (10)). For this we need one more lemma whose proof, which we omit, can be done in the same way as in the scalar case (cf. e.g., [8]).

Lemma 3.1. Let $f, g \in W_{\infty}(H)$. Then $(f, g)_{H} \in W_{\infty}$ and

$$
L(f, g)=(L f, g)+(f, L g)+(\nabla f, \nabla g)_{H \otimes H} .
$$

By this lemma we have

$$
\begin{aligned}
& L^{2}\left(\sum_{i=0}^{2^{n}-1}\left(M_{t_{i+1}^{n} \wedge t}-M_{t^{n} \wedge t}\right)^{2}\right) \\
& =\sum_{i=0}^{2^{n}-1}\left\{2 \left(\left(M_{t_{i+1}^{n} \wedge t}-M_{t_{i^{n} \wedge t}}\right) L^{2}\left(M_{t_{i+1}^{n} \wedge t}-M_{t^{n} \wedge t}\right)+\left(L\left(M_{t_{i+1}^{n} \wedge t}-M_{t_{i}^{n} \wedge t}\right)\right)^{2}\right.\right. \\
& +\left(\nabla\left(M_{t_{i+1}^{n} \wedge t}-M_{t_{i}^{n} \wedge t}\right), \nabla L\left(M_{t_{i+1}^{n} \wedge t}-M_{t_{i} \wedge}\right)\right)_{H} \\
& \left.+\left(L D\left(M_{t_{i+1}^{n} \wedge t}-M_{t^{n} \wedge t}\right), D\left(M_{t_{i+1}^{n} \wedge t}-M_{t^{n} \wedge t}\right)\right)_{H}\right) \\
& \left.+\left\|\nabla^{2}\left(M_{t_{i+1}^{n} \wedge t}-M_{t^{n} \wedge t}\right)\right\|_{H \otimes H}\right\}
\end{aligned}
$$




$$
\triangleq \sum_{j=1}^{5} J_{j}^{n}
$$

The estimation of $J_{j}^{n}(j=1,2,3)$ being done in a way similar to that of $I_{1}{ }^{n}$ and $I_{2}{ }^{n}$, we first have

$$
\sup _{n, t} E\left(\left|J_{1}{ }^{n}\right|^{p}+\left|J_{2}{ }^{n}\right|^{p}+\left|J_{3}{ }^{n}\right|^{p}\right)<\infty .
$$

For $J_{4}{ }^{n}$ we write

$$
\begin{aligned}
\left|J_{4}{ }^{n}\right| \leq & \sum_{i=0}^{2^{n}-1}\left\|L D\left(M_{t_{i+1}^{n} \wedge t}-M_{t_{i}{ }^{n} \wedge t}\right)\right\|_{H}^{2} \\
& +\sum_{i=0}^{2^{n}-1}\left\|\nabla\left(M_{t_{i+1}^{n} \wedge t}-M_{t^{n} \wedge t}\right)\right\|_{H}^{2} \\
\triangleq & J_{41}^{n}+J_{42}^{n} .
\end{aligned}
$$

$J_{42}^{n}$ will cause no problem, as we have seen above. To estimate $J_{41}^{n}$ we first make the following observation:

$$
\begin{aligned}
& L D\left(M_{t_{i+1}^{n} \wedge t}-M_{t_{i}^{n} \wedge t}\right) \\
& \quad=\sum_{j=1}^{d} \int_{t_{i}^{n} \wedge t}^{t_{i+1}^{n} \wedge t}\left(L \nabla f_{u}^{j}-\frac{1}{2} \nabla f_{u}^{j}\right) d w_{u}{ }^{j}+\int_{t_{i} n \wedge t \wedge .}^{t_{i+1}^{n} \wedge t \wedge \bullet} L f_{u} d u .
\end{aligned}
$$

Then we obtain

$$
\begin{aligned}
& \sum_{i=0}^{2^{n}-1}\left(\left\|L D\left(M_{t_{i+1}^{n} \wedge t}-M_{t^{n} \wedge}\right)\right\|_{H}^{2}\right) \\
& \quad \leq \sum_{j=1}^{d} \sum_{i=0}^{2^{n}-1}\left(\left\|\int_{t_{i}^{n} \wedge t}^{t_{i+1}^{n} \wedge t}\left(L D f_{u}^{j}-\frac{1}{2} \nabla f_{u}^{j}\right) d w_{u}^{j}\right\|_{H}^{2}+\left\|\int_{t_{i} i^{n} \wedge t \wedge}^{t_{i+1}^{n} \wedge t \wedge \cdot} L f_{u} d u\right\|_{H}^{2}\right) .
\end{aligned}
$$

But the RHS can be estimated in the same way as for $I_{2}{ }^{n}$, so we have

$$
\sup _{n, t} E\left|J_{41}^{n}\right|^{p}<\infty
$$

Finally for $J_{5}{ }^{n}$ we use the Proposition 3.1 to obtain

$$
\begin{aligned}
J_{5}{ }^{n} & =\sum_{i=0}^{2^{n}-1}\left(\left\|\sum_{j=1}^{d} \int_{t_{i}^{n} \wedge t}^{t_{i^{n} \wedge}^{n} \wedge t} \nabla^{2} f_{u}^{j} d w_{u}^{j}+\int_{t^{n} \wedge t \wedge \cdot}^{t_{i+1}^{n} \wedge t \wedge \cdot} \nabla f_{u} d u\right\|_{H \otimes H}^{2}\right) \\
& \leq C \sum_{i=0}^{2^{n}-1}\left(\sum_{j=1}^{d}\left\|\int_{t_{i}^{n} \wedge t}^{t_{i+1}^{n} \wedge t} \nabla^{2} f_{u}^{j} d w_{u}^{j}\right\|_{H \otimes H}^{2}+\left\|\int_{t^{n} \wedge t \wedge \cdot}^{t_{i+1}^{n} \wedge t \wedge \cdot} \nabla f_{u} d u\right\|_{H \otimes H}^{2}\right),
\end{aligned}
$$

which can also be estmated in exactly the same way as for $I_{2}{ }^{n}$. Therefore

$$
\sup _{n, t} E\left|J_{5}{ }^{n}\right|^{p}<\infty
$$


Combining (17) (18) (19) (20) (21) we get

$$
\sup _{n, t}\left\|\sum_{i=0}^{2^{n}-1}\left(M_{t_{i+1}^{n} \wedge t}-M_{t^{n} \wedge t}\right)^{2}\right\|_{p, 4}<\infty
$$

proving (10) for $r=2$. Doing the same thing for higher order derivatives, step by step, we can complete the proof of (10).

Now the proof of Theorem 3.2 is finished.

Q.E.D.

\section{Two-parameter smooth martingales}

Different to the one-parameter case, a two-parameter Brownian martingale does not always admit a representation of an ordinary Ito stochastic integral in the plane: some additional term called stochastic integral of the second type, which seems to be more difficult to handle, especially when Malliavin calculus is involved, appears in the representation. It is for this reason that we restrict ourselves in this section to the case of strong martingales, which admit always representation of stochastic integrals of the first type, i.e., the ordinary Ito integrals in the plane.

We still start by introducing some notions and notations. Parallel to the one-parameter case, we now work on the probability space $X=C_{0}([0,1] \times[0$, 1] $\rightarrow \boldsymbol{R}^{d}$ ), together with its Cameron-Martin subspace

$$
H=\left\{f \in X, \frac{\partial^{2} f}{\partial s \partial t} \text { exists a.e. and } \int_{0}^{1} \int_{0}^{1}\left\|\frac{\partial^{2} f(s, t)}{\partial s \partial t}\right\|_{R^{d}} d s d t<\infty\right\}
$$

and the two-parameter standard Wiener measure $\mu$ defined on the Borel $\sigma$ algebra $\mathscr{I}$ of $X$. Let $\{w(s, t), 0 \leq s, t \leq 1\}$ be the coordinate Brownian sheet on $X$ and $\mathscr{I}_{s, t}$ the sub- $\sigma$-algebra of $\mathscr{I}$ generated by the Brownian paths up to time $(s, t)$. Then any $\left\{\mathscr{F}_{s, t}\right\}$-strong continuous $L^{2}$-martingale has a representation (cf. [3], [11])

$$
M_{s, t}=\sum_{j=1}^{d} \int_{0}^{t} \int_{0}^{s} f_{u, v}^{j} d w_{u, v}^{j}, \quad 0 \leq s, t \leq 1 .
$$

With the same notations in Malliavin caculus used in section 1, we can state the following

Definition 4.1. We say that $\boldsymbol{M}=\left\{M_{s, t}, 0 \leq s, t \leq 1\right\}$ is a two-parameter smooth martingale if

(C2) $f_{u, v}^{j} \in W_{\infty}\left(\boldsymbol{R}^{d}\right)$, for almost all $0 \leq u, v \leq 1$ and $\int_{0}^{1} \int_{0}^{1}\left\|f_{u, v}\right\|_{p, 2 r}^{p} d u d v<\infty$ $\forall p, r$.

Analogue to [9, Theorem 4.2], we have 
Theorem 4.1, Given a two parameter smooth martingale $M$ represented as (22), then

(i) $M_{s, t} \in W_{\infty}\left(\boldsymbol{R}^{d}\right)$, for all $0 \leq s, t \leq 0$.

(ii) There exists a decreasing sequence $\left\{O_{n}, n \geq 1\right\}$ of open subsets of $X$ and a function $\tilde{M}:\left(\cup_{n \geq 1} O_{n}^{c}\right) \times[0,1]^{2} \rightarrow \boldsymbol{R}^{d}$ such that

(a) $\tilde{M}$ is continuous on $O_{n}{ }^{c} \times[0,1]^{2}$, for each $n \geq 1$.

(b) $C_{p, r}\left(O_{n}\right) \rightarrow 0$ as $n \rightarrow \infty$ for all $p, r$.

(c) $\tilde{M}_{s, t}=M_{s, t}$ almost surely, for all $0 \leq s, t \leq 0$.

Proof. Though the proof is similar to that of [9, Theorem 4.2], we give it for completeness. First we have by Stroock's commutation formula:

$$
L\left(\sum_{j=1}^{d} \int_{0}^{t} \int_{0}^{s} f_{u, v}^{j} d w_{u, v}^{j}\right)=\sum_{j=1}^{d}\left\{\int_{0}^{t} \int_{0}^{s}\left(L f_{u, v}^{j}-\frac{1}{2} f_{u, v}^{j}\right) d w_{u, v}^{j}\right\}
$$

Hence by Burkholder's inequality for two-parameter martingales (cf. [15]), we obtain

$$
\sup _{s, t}\left\|\sum_{j=1}^{d} \int_{0}^{t} \int_{0}^{s} f_{u, v}^{j} d w_{u, v}^{j}\right\|_{p, 2}^{p} \leq \int_{0}^{1} \int_{0}^{1}\left(\sum_{j=1}^{d}\left\|f_{u, v}^{j}\right\|_{p, 2}^{p}\right) d u d v<\infty, \forall p>1 ;
$$

and in the same way

$$
\sup _{s, t}\left\|\sum_{j=1}^{d} \int_{0}^{t} \int_{0}^{s} f_{u, v}^{j} d w_{u, v}^{j}\right\|_{p, r}^{p} \leq \int_{0}^{1} \int_{0}^{1}\left(\sum_{j=1}^{d}\left\|f_{u, v}^{j}\right\|_{p, r}^{p}\right) d u d v<\infty
$$

for all $p, r$. In particular, (i) holds. Using again Burkholder's inequality we obtain for $p \geq 2, s \leq s^{\prime}, t \leq t^{\prime}$ :

$$
\begin{aligned}
& \left\|M_{s, t}-M_{s^{\prime}, t^{\prime}}\right\|_{p, 2}^{p} \\
& \quad \leq C E\left\{\sum_{j=1}^{d} \int_{s}^{s^{\prime}} \int_{t}^{t^{\prime}}\left|L f_{u, v}^{j}-\frac{1}{2} f_{u, v}^{j}\right|^{2} d u d v\right\}^{p / 2} \\
& \quad \leq C\left(\left|t^{\prime}-t\right|\left|s^{\prime}-s\right|\right)^{p / 2-1}\left(\sum_{j=1}^{d} \int_{s}^{s^{\prime}} \int_{t}^{t^{\prime}}\left\|f_{u, v}^{j}\right\|_{p, 2}^{p} d u d v\right) \\
& \quad \leq C\left(\left|t^{\prime}-t\right|^{p-2}+\left|s^{\prime}-s\right|^{p-2}\right) .
\end{aligned}
$$

And in general we can prove that

$$
\left\|M_{s, t}-M_{s^{\prime}, t^{\prime}}\right\|_{p, r}^{p} \leq C\left[\left|t^{\prime}-t\right|^{p-2}+\left|s^{\prime}-s\right|^{p-2}\right]
$$

for all $r>0$. Therefore Theorem 3.1 and Lemma 4.1 of [16] apply and the proof is complete.

Q.E.D.

The following result is the counterpart of Theorem 3.1, and we omit its 
proof.

Theorem 4.2. $\langle M\rangle=\left\{\langle M\rangle_{s, t} 0 \leq s, t \leq 1\right\}$ admits an $\infty$-modification.

We still denote by $\boldsymbol{M}$ (resp. $\langle M\rangle)$ itself its $\infty$-modification $\tilde{\boldsymbol{M}}$ (resp. $\langle\tilde{\boldsymbol{M}}\rangle$ ). Our main result in this section is the following counterpart of Theorem 3.2.

Theorem 4.3. We have the following convergence:

$$
\lim _{n \rightarrow \infty} \sum_{i=0}^{2^{n}-1} \sum_{j=0}^{2^{n}-1}\left(M\left(\Delta_{i j}^{n}(s, t)\right)\right)^{2}=\sum_{k=1}^{d} \int_{0}^{s} \int_{0}^{t} f_{u, v}^{k^{2}} d u d v
$$

uniformly in $(s, t) \in[0,1]^{2}$, q.s., where $\Delta_{i j}^{n}(s, t)=\left[t_{i}^{n} \wedge t, t_{i+1}^{n} \wedge t\right) \times\left[s_{i}^{n} \wedge s, s_{i+1}^{n} \wedge\right.$ $s)$ and $M\left(\Delta_{i j}^{n}(s, t)\right)=M_{t_{i+1}^{n} \wedge t, s_{j+1}^{n} \wedge s}-M_{t_{i}^{n} \wedge t, s_{j+1}^{n} \wedge s}-M_{t_{i+1}^{n} \wedge t, s_{j}^{n} \wedge s}+M_{t_{i}^{n} \wedge t, s_{j}{ }^{n} \wedge s}$.

Proof. The idea of the proof remains the same as that of Theorem 3.2, though the calculus is more delicate. This is why we do not give all the details below. Our effort will be concentrated only on those terms which didn't appear in the proof of Theorem 3.2. First, note that, as in Theorem 3.2, we should define a random field parametrized by $[0,1]^{3}$ as follows:

$$
X(\xi, s, t)= \begin{cases}X\left(2^{-n}, s, t\right)+\left(\xi-2^{-n}\right)\left(2^{-n}-2^{-(n+1)}\right)^{-1} \\ \quad \times\left(X\left(2^{-n}, s, t\right)-X\left(2^{-(n+1)}, s, t\right)\right) & \text { if } 2^{-(n+1)} \leq \xi \leq 2^{-n}, \\ \sum_{k=1}^{d} \int_{0}^{s} \int_{0}^{t} f_{u, v}^{k^{2}} d u d v & \text { if } \xi=0 .\end{cases}
$$

where

$$
X\left(2^{-n}, s, t\right)=\sum_{i=0}^{2^{n}-1} \sum_{j=0}^{2^{n}-1}\left(M\left(\Delta_{i j}^{n}(s, t)\right)\right)^{2} .
$$

Then we can reduce the proof to proving the following facts:

$$
\begin{aligned}
& \sup _{\xi}\left\|X(\xi, s, t)-X\left(\xi, s^{\prime}, t^{\prime}\right)\right\|_{p}^{p} \leq C\left|s-s^{\prime}\right|^{\frac{p-1}{2}}+C\left|t-t^{\prime}\right|^{\frac{p-1}{2}} \\
& \sup _{s, t}\left\|X\left(2^{-n}, s, t\right)-X(0, s, t)\right\|_{p}^{p} \leq C 2^{-\frac{n(p-1)}{2}} \\
& \sup _{s, t}\|X(s, t)\|_{p, 2 r}<\infty
\end{aligned}
$$

Proof of (24). Put

$$
\begin{aligned}
N^{k, l}\left(\Delta_{i j}^{n}(s, t)\right)= & \iiint \int_{\Delta_{i j}^{n}(s, t)^{2}} \chi\left(u_{1}, v_{1} ; u_{2}, v_{2}\right) \\
& \times f_{u_{1}, v_{1}}^{k} f_{u_{2}, v_{2}}^{l} d w_{u_{1}, v_{1}}^{k} d w_{u_{2}, v_{2}}^{l},
\end{aligned}
$$

where 


$$
\chi\left(u_{1}, v_{1} ; u_{2}, v_{2}\right)= \begin{cases}0 & \text { if }\left(u_{1}, v_{1}\right) \leq\left(u_{2}, v_{2}\right) \text { or } \quad\left(u_{2}, v_{2}\right) \leq\left(u_{1}, v_{1}\right) \\ 1 & \text { otherwise }\end{cases}
$$

Then by Ito's formula for two-parameter processes (cf. [3], [11], [14]) we have

$$
\begin{aligned}
\sup _{s, t} & \left\|X\left(2^{-n}, s, t\right)-X(0, s, t)\right\|_{p}^{p} \\
= & \sup _{s, t}\left\|\sum_{i=0}^{2^{n}-1} \sum_{j=0}^{2^{n}-1}\left\{M\left(\Delta_{i j}^{n}(s, t)\right)^{2}-\iint_{\Delta_{i j}^{n}(s, t)} \sum_{k=1}^{d} f_{u, v}^{k^{2}} d u d v\right\}\right\|_{p}^{p} \\
= & \sup _{s, t} \| \sum_{i=0}^{2^{n}-1} \sum_{j=0}^{2^{n}-1}\left\{\sum_{k=1}^{d} \int_{\Delta_{i j}^{n}(s, t)} 2 M\left(\Delta_{i j}^{n}(s, t)\right) f_{u, v}^{k} d w_{u, v}^{k}\right. \\
& \left.+\sum_{k=1}^{d} \sum_{l=1}^{d} N^{k, l}\left(\Delta_{i j}^{n}(s, t)\right)\right\} \|_{p}^{p} \\
\leq & C \sup _{s, t} \sum_{k=1}^{d}\left\{\left\|\sum_{i=0}^{2^{n}-1} \sum_{j=0}^{2^{n}-1} \iint_{\Delta_{i j}^{n}(s, t)} M\left(\Delta_{i j}^{n}(s, t)\right) f_{u, v}^{k} d w_{u, v}^{k}\right\|_{p}^{p}\right. \\
& \left.+\left\|\sum_{k=1}^{d} \sum_{l=1}^{d} N^{k, l}\left(\Delta_{i j}^{n}(s, t)\right)\right\|_{p}^{p}\right\} \\
\triangleq & C \sum_{k=1}^{d}\left\{\left\|K_{1}\right\|_{p}^{p}+\left\|K_{2}\right\|_{p}^{p}\right\} .
\end{aligned}
$$

For $K_{1}$, we use Burkholder's inequality for both two-parameter martingales with discrete parameter (cf. [10]) and continuous parameter (cf. [15]) to obtain, in the same way as in the last section,

$$
E\left|K_{1}\right|^{p} \leq C 4^{-n(p / 2-1)}
$$

For $K_{2}$ we use again Burkholder's inequality and the properties of double stochastic integrals to obtain

$$
\begin{aligned}
& E\left|K_{2}\right|^{p} \\
& \leq C \sum_{k=1}^{d} \sum_{l=1}^{d} E\left\{\sum_{i=0}^{2^{n}-1} \sum_{j=0}^{2^{n}-1} N^{k, l}\left(\Delta_{i j}^{n}(s, t)\right)^{2}\right\}^{p / 2} \\
& \leq C 4^{n(p / 2-1)} \sum_{k=1}^{d} \sum_{l=1}^{d} \sum_{i=0}^{2^{n}-1} \sum_{j=0}^{2^{n-1}} E\left|N^{k, l}\left(\Delta_{i j}^{n}(s, t)\right)\right|^{p} \\
& =C 4^{n(p / 2-1)} \sum_{k=1}^{d} \sum_{l=1}^{d} \sum_{i=0}^{2^{n}-1} \sum_{j=0}^{2^{n}-1} E \mid \iiint \int_{\Delta_{i j}^{n}(s, t) 2} \chi\left(u_{1}, v_{1} ; u_{2}, v_{2}\right) \\
& \quad \times\left. f_{u_{1}, v_{1}}^{k} f_{u_{2}, v_{2}}^{l} d w_{u_{1}, v_{1}}^{k} d w_{u_{2}, v_{2}}^{l}\right|^{p}
\end{aligned}
$$




$$
\begin{aligned}
\leq & C 4^{n(p / 2-1)} 16^{-n(p / 2-1)} \sum_{k=1}^{d} \sum_{l=1}^{d} \sum_{i=0}^{2^{n}-1} \sum_{j=0}^{2^{n}-1} E \iiint \int_{\Delta_{i j}^{n}(s, t)^{2}} \\
& \times\left|f_{u_{1}, v_{1}}^{k} f_{u_{2}, v_{2}}^{l}\right|^{p} d u_{1} d v_{1} d u_{2} d v_{2} \\
\leq & C 4^{-n(p / 2-1)} .
\end{aligned}
$$

Combining (26), (27) and (28) we get (24).

Proof of (23) and (25). The proof of (23) and (25) is completely similar to that of (4) and (5), since now we needn't to use Ito formula and so no term with double stochastic integrals will appear (of course we should use Burkholder's inequality for two-parameter martingales instead of that for oneparameter martingales). We don't give the details. This completes the proof.

Q.E.D.

\section{Remarks and Applications}

In this final section we would like first to remark that the above results can extend to mutual quadratic variation of so-called smooth semimartingales. To be precise, let $S_{1}$ and $S_{2}$ be two smooth semi-martingales of the form

$$
S_{l}(t)=\sum_{j=1}^{d} \int_{0}^{t} a_{s}{ }^{l j} d w_{s}^{j}+\sum_{j=1}^{d} \int_{0}^{t} b_{s}^{l j} d s, \quad l=1,2, \quad t \in[0,1] .
$$

Here $a$ and $b$ satisfy the condition (C1). Then the techniques used above allow to prove that

$$
\begin{aligned}
& \lim _{n \rightarrow \infty} \sum\left(S_{1}\left(t_{i+1}^{n} \wedge t\right)-S_{1}\left(t_{i}^{n} \wedge t\right)\right)\left(S_{2}\left(t_{i+1}^{n} \wedge t\right)-S_{2}\left(t_{i}{ }^{n} \wedge t\right)\right) \\
& \quad=\sum_{j=1}^{d} \int_{0}^{t} a_{s}{ }^{1 j} a_{s}{ }^{2 j} d s
\end{aligned}
$$

uniformly in $t \in[0,1]$, q.s.

Now we consider the case of stochastic differential equations. Suppose we are given functions $\sigma \in C_{b}{ }^{\infty}\left(\boldsymbol{R}^{m} \rightarrow \boldsymbol{R}^{m} \otimes \boldsymbol{R}^{d}\right)$ and $b \in C_{b}{ }^{\infty}\left(\boldsymbol{R}^{m} \rightarrow \boldsymbol{R}^{m}\right)$. It was proved in [16] that the unique solution to the following SDE

$$
\left\{\begin{array}{l}
d x(t)=\sigma(x(t)) d w(t)+b(x(t)) d t \\
x(0)=x_{0}
\end{array}\right.
$$

has an $\infty$-modification. We will denote it by $x(t)=\left(x^{1}(t), \cdots, x^{m}(t)\right)$. It is easily seen that every $x^{k}(k=1, \cdots, m)$ is a smooth semimartingale. Therefore we have by (29) 


$$
\lim _{n \rightarrow \infty} \sum\left(x_{t_{i+1}^{n} \wedge t}-x_{t_{i}^{n} \wedge t}\right)\left(x_{t_{i+1}^{n} \wedge t}-x_{t_{i}^{n} \wedge t}\right)^{*}=\int_{0}^{t} \sigma(x(s)) \sigma^{*}(x(s)) d s
$$

uniformly in $t \in[0,1]$, q.s. In particular, in the case $\sigma \equiv I_{d}, b \equiv 0$, where $I_{d}$ is the $d \times d$ identity matrix, we get

$$
\lim _{n \rightarrow \infty} \sum\left(w_{t_{i+1}^{n} \wedge t}-w_{t_{i}^{n} \wedge t}\right)\left(w_{t_{i+1}^{n} \wedge t}-w_{t_{i}^{n} \wedge t}\right)^{*}=t I_{d}
$$

uniformly in $t \in[0,1]$, q.s. It is remarkable that (30) establishes one more quasi sure property of Brownian motion, of which several quasi sure properties have been already established (cf. [19]). In the context of Dirichlet capacity over Wiener space, i.e., in the case $(p, r)=(2,1),(30)$ was established by Fukushima ([28]) using a different method (see also the recent book of Bouleau-Hirsch [1]). Finally it is needless to say that all the above statements have also their analogues in the two-parameter case.

Acknowledgement. The author is very grateful to Professor S. Watanabe and Professor I. Shigekawa for useful discussions.

Department of Mathematics Huazhong University of Science and Technology Wuchang, Hubei 430074

P. R. China

and

Department of Mathematics Kyoto University

\section{References}

[1] N. Bouleau, and F. Hirsch, Dirichlet Forms and Analysis on Wiener Space, de Gruyter Studies in Math., vol. 14, Berlin. New York, (1990).

[2] D. L. Burkholder, A geometrical characterization of Banach spaces in which martingales difference sequences are unconditional, Ann. Probab., 9(1981), 997-1011.

[ 3 ] R. Caroli, and J. B. Walsh, Stochastic integrals in the plane, Acta Math., 134 (1975), 111-183.

[ 4 ] L. Chevalier, Martingales continues à deux parametres, Bull. Sci. Math. (2), 106 (1982), 19-62.

[5] Z. Huang, Foundation of Stochastic Analysis, Wuhan Univ. Press, 1988 (in Chinese).

[6] S. Kusuoka, and D. Stroock, Applications of the Malliavin calculus. Part I, Taniguchi Intern. Symp. SA. Katata (1982), 271-306, Kinokunia, Tokyo, 1983.

[ 7 ] P. Malliavin, Implicit functions in finite corank on the Wiener space, Taniguchi Intern. Symp. SA. Katata (1982), 369-386, Kinokunia, Tokyo, 1983.

[ 8 ] P. Malliavin, Differential Analysis in Stochastic Analysis, Cours MIT.

[ 9 ] P. Malliavin, and D. Nualart, Quasi sure analysis of stochastic flows and Banach space valued smooth functionals on the Wiener space, J. Funct. Anal., 112 (1993), 287-317.

[10] P. A. Meyer, Théorie élémentaire des processus à deux indices, Lecture Notes in Math 863 (1981), 1-39.

[11] D. Michel, Formule de Stokes Stochastique, Bull. Sci. Math. (2), 103 (1979), 193-240.

[12] P. W. Millar, Martingales integrals, Trans. Amer. Math. Soc., 133 (1968), 145-166. 
[13] D. Nualart, On the quadratic variation of two-parameter continuous martingales, Ann. Probab., 12 (1984), 445-457.

[14] D. Nualart, Une formule d'lto pour les martingales continues à deux indices et quelques applications, Ann. Inst. H. Poincaré, 20 (1984), 251-275.

[15] D. Nualart, Variation quadratiques et Inégalités pour les martingales à deux indices, Stochastics, 15 (1985), 51-63.

[16] J. Ren, Analyse quasi sûre des équation différentielles stochastiques, Bull Sci. Math (2), 114 (1990), 187-213.

[17] J. Ren, On smooth martingales, to appear in J. F. A.

[18] I. Shigekawa, Sobolev space of Banach-valued functions associated with a Markov process, preprint (1992).

[19] M. Takeda, (r, p)-capacities on the Wiener space and properties of Brownian motion, $Z$. Wahrsch. Verw. Gebiete, 68 (1984), 149-162.

[20] S. Watanabe, Stochastic Differential Equations and Malliavin Calculus, Tata Inst. Fund. Research (bombay). vol. 73 Springer-Verlag, 1984.

[21] J. Ren, Analyse quasi sûre des martingales régulières, to appear in C. R. Acad. Sci. Paris (1993).

[22] M. Fukushima, Basic properties of Brownian motion and capacity on the Wiener space, J. Math. Soc. Japan, 36 (1984), 161-176. 